\title{
ON THE COINCIDENCE SET IN VARIATIONAL INEQUALITIES
}

\author{
HANS LEWY
}

Variational inequalities, i.e., problems of the calculus of variations where inequalities limit the space of competing functions, have received much attention in recent years. A large literature, too numerous to be listed in this note, deals with existence and smoothness of solutions in their dependence on the smoothness of the bounding inequalities. An attractive feature of these problems lies in that the set of points where these inequalities become essential for the solution is not known a priori. The skill of the workers provides a setting of function spaces such that the distinction between points, where the competing function is "free" and its variation is encumbered, does not affect its smoothness; shows existence of a solution in this space and then proceeds to prove a higher degree of smoothness, again by arguments which do not necessitate a precise knowledge of the nature of the set $S$ where the bounding inequalities become effective. Very little indeed is known about this set $S$ : it does in general not depend continuously on the data of the problem; in a few cases under strong convexity assumptions a certain simplicity of $S$ has been ascertained.

The elusiveness of the nature of $S$ is the challenge which motivated the present paper, to be considered a sequal to [1]. We are concerned with only the simplest nontrivial problem of variational inequalities, but succeed in clarifying the topological character of $S$ in this case.

For the sake of brevity and self-sufficiency we have chosen to speak here only of properties of certain superharmonics without elaborating their wellknown connection with the Dirichlet integral which actually provides the variational background described in the foregoing introduction.

1. Lemma 1.1. Let $(A, B)$ be an open interval of $\boldsymbol{R}^{1}$ and contain the compact support $S$ of a measure $\mu$ with $\mu(S)>0$. Let $u(x)=\int_{S}-\log |x-y| d \mu(y)$, $x \in \boldsymbol{R}^{1}$, and suppose the existence of a $C^{1}[A, B]$ function $f(x)$ such that

$$
f(x) \leq u(x), \quad x \in(A, B) ; \quad f(x)=u(x), \quad x \in S .
$$

Then $\mu$ is absolutely continuous in $(A, B)$.

Proof. It was shown in [1] that $u(x) \in C^{1}(A, B)$. Extend $u(x)$ continuously into the upper half of a $z$-plane as harmonic function by

\footnotetext{
Received January 10, 1972.
} 


$$
u(z)=\int_{S}-\log |z-y| d \mu(y) .
$$

A conjugate harmonic is

$$
v(z)=\int_{S}-\theta(z-y) d \mu(y)
$$

where $\theta(z-y)$ is the angle of the vector $z-y$ with the positive direction of $\boldsymbol{R}^{1}$. As $z$ tends to a point $x$ of $\boldsymbol{R}^{1}, v(z)$ tends to $-\pi \int_{x}^{B} d \mu(y)$, which is a continuous function of $x$ since the continuity of $u(x)$ forces $\mu(x)$, the measure of any single point $x$, to vanish. $u(z)+i v(z)=F(z)$ is holomorphic for $z$ in the upper half plane, continuous on its closure, with boundary values on $\boldsymbol{R}^{1}$ which are of bounded variation on every compact portion of $\boldsymbol{R}^{1}$ such as $[A, B]$. A variant of F. and M. Riesz' theorem applies: these boundary values are absolutely continuous on every compact portion of $\boldsymbol{R}^{1}$. In fact, take a $C_{0}^{\infty}(A, B)$ function $p(x)$ of compact support with $p(x) \geq 0$ and $\int_{-\infty}^{\infty} p(y) d y=\mu(S)$ and set

$$
F_{1}(z)=\int_{-\infty}^{\infty}-\log (z-y) p(y) d y
$$

The difference $F(z)-F_{1}(z)$ is holormorphic in the upper half plane and assumes continuous boundary values on $\boldsymbol{R}^{1}$. Integration by parts yields that $F(z)$ $-F_{1}(z) \rightarrow 0$ as $z \rightarrow \infty$ and, if $\operatorname{Re} z<A$ or $\operatorname{Re} z>B$, that $\left|F^{\prime}(z)-F_{1}^{\prime}(z)\right| \leq$ $\mu(S)|| z-\left.B\right|^{-2}-|z-A|^{-2} \mid$. Hence $\int_{-\infty}^{\infty}\left|d\left(F(x)-F_{1}(x)\right)\right|<\infty$, so that $F(z)$ $-F_{1}(z)$ may be regarded, through a conformal map of the upper half $z$-plane on the unit disc of a $\zeta$-plane, as holomorphic function of $\zeta$ with boundary values of bounded variation. By $\mathrm{F}$. and $\mathrm{M}$. Riesz these are absolutely continuous. Hence $F(x)-F_{1}(x)$ is absolutely continuous as function of $x$ on any bounded portion of $R^{1}$ in view of the boundednes of $|d z / d \zeta|$ on that portion. Moreover, $F_{1}^{\prime}(x)$ is continuous, hence $F(x)$, in particular $\operatorname{Im} F(x)=-\pi \int_{x}^{B} d \mu(y)$, is absolutely continuous.

We write $F^{\prime}(z)=U(z)+i V(z) \cdot \operatorname{Re} F^{\prime}(z)=U(z)$ is continuous on $\operatorname{Im} z \geq 0$ by the quoted theorem of [1], and tends to zero as $z \rightarrow \infty$. Therefore there is an $M$ with $M \geq|U(z)|$. Put, with the above meaning of $\zeta$,

$$
F^{\prime}(z)=\mathscr{G}(\zeta)=\tilde{U}(\zeta)+i \tilde{V}(\zeta)
$$

$F^{\prime}(z)(d \zeta / d z)$ is holomorphic in $\operatorname{Im} z>0$; the primitive function 


$$
\int F^{\prime}(z) \frac{d \zeta}{d z} d z=F(z) \frac{d \zeta}{d z}-\int F(z) \frac{d^{2} \zeta}{d z^{2}} d z
$$

is readily seen to be bounded and continuous in $\operatorname{Im} z \geq 0$. This means that $G_{1}(\zeta)=\int(G(\zeta)-G(0)) \mathrm{d} \zeta / \zeta$ is continuous in $|\zeta| \leq 1$, and $G_{1}(\zeta)=P_{G_{1}}(\zeta)$, the Poisson integral of the boundary values of $G_{1}$; consequently $G(\zeta)=P_{G}(\zeta)$ if $|\zeta|<1$. Hence $G(\zeta)$ is a holomorphic function of $\zeta$ in $|\zeta|<1$, representable asPoisson integral of its almost everywhere existing (normal) boundary values. Such a function is said to belong to the Hardy class $H^{1}$.

2. Lemma 2.1. $G^{2}(\zeta)$ belongs to $H^{1}$.

Proof. For $0<r<1$,

$$
\int_{0}^{2 \pi} G^{2}\left(r e^{i \varphi}\right) d \varphi=2 \pi G^{2}(0)=\int_{0}^{2 \pi}\left(\tilde{U}^{2}-\tilde{V}^{2}+2 i \tilde{U} \tilde{V}\right)\left(r e^{i \varphi}\right) d \varphi=c_{1}+i c_{2}
$$

with real $c_{j}$. Since $|\tilde{U}| \leq M$,

$$
\int_{0}^{2 \pi} \tilde{V}^{2}\left(r e^{i \varphi}\right) d \varphi=-c_{1}+\int_{0}^{2 \pi} \tilde{U}^{2} d \varphi \leq-c_{1}+2 \pi M^{2}
$$

Thus $\int G^{2}(\zeta) d \zeta$ is bounded in $|\zeta|<1$ and of uniformly bounded variation on all circles $|\zeta|=r$. Therefore $G_{2}(\zeta)=\int\left(G^{2}(\zeta)-G^{2}(0)\right) d \zeta / \zeta=P_{G_{2}}(\zeta), G^{2}(\zeta)=$ $P_{G^{2}}(\zeta)$, by F. and M. Riesz. The boundary values of $G^{2}$ exist almost everywhere and thus are given by those of $\tilde{U}^{2}-\tilde{V}^{2}+2 i \tilde{U} \tilde{V}$.

3. Theorem 3.1. Let $\mu$ be a measure of compact support $S$ contained in an interval $(A, B)$ of $\boldsymbol{R}^{1}, \mu(S)>0$, and $u(x)=\int_{S}-\log |x-y| d \mu(y)$ its logarithmic potential. Suppose there exists a real function $f(x)$, analytic in $[A$, $B]$ and such that

$$
u(x) \geq f(x), \quad x \in(A, B) ; \quad u(x)=f(x), \quad x \in S .
$$

Then $S$ is the union of finitely many disjoint intervals.

Proof. There is a neighborhood $N$ of $(A, B)$ in the $z$-plane into which $f(x)$ can be extended as holomorphic function $f(z)$. Consider

$$
\left(F^{\prime}(z)-f^{\prime}(z)\right)^{2}=(U+i V-d f / d z)^{2}
$$

which is holomorphic in $N \cap\{z: \operatorname{Im} z>0\}$ and assumes on $(A, B)$ almost everywhere the boundary values

$$
(U(x)-d f / d x)^{2}-V^{2}(x)+2 i(U-d f / d x) V .
$$


The primitive function $\int_{A}^{z}\left(F^{\prime}(z)-f^{\prime}(z)\right)^{2} d z$ has on $(A, B)$ the continuous boundary values

$$
\int_{A}^{x}\left(\left(U-d f / d x^{\prime}\right)^{2}-V^{2}\right) d x^{\prime}+2 i \int_{A}^{x}\left(U-d f / d x^{\prime}\right) V d x^{\prime},
$$

on account of Lemma 2.1. Now the second integral vanishes on $(A, B)$ since $U\left(x^{\prime}\right)=d u / d x=d f / d x$ for $x^{\prime} \in S$, and $V\left(x^{\prime}\right)=d v / d x=0$ a.e. for $x^{\prime} \in(A, B)-S$. It follows that the first integral is analytically extensible into a plane neighborhood of $(A, B)$. In particular, it is analytic and has an analytic derivative denoted by $g(x)$, and

$$
g(x)=(U-d f / d x)^{2}-V^{2}(x)
$$

almost everywhere on $(A, B)$. Accordingly we may, if necessary, redefine $V(x)$ on a set of measure zero, so as to make $-V(x)$ continuous and $\geq 0$, since we know that $U(x), d f / d x, g(x)$ are continuous. This improved $V(x)$ has the same integral $v(x)$ as before and can be substituted in all relations involving integration.

$g(x)$ is not identically zero, for on $S$ we have $g(x)=-V^{2}(x)$ and we know $\pi \int_{S} V(x) d x=-\mu(S) \neq 0$. As $-V(x)>0$ a.e. on $S$, we have $g(x)<0$ on $S$ excepting finitely many points ( $g$ being analytic). Conversely, if $g(x)<0$, then certainly $V^{2}(x) \neq 0,-V(x)>0, x \in S$. It follows that $S$ is the union of disjoint intervals at whose endpoints $g(x)$ changes sign, and we have the formulas

$$
\begin{aligned}
-V(x) & =\sqrt{-g(x)}, x \in S ; & V(x) & =0, x \in(A, B)-S, \\
U(x) & =d f / d x, x \in S ; & & U(x)=d f / d x \pm \sqrt{g(x)}, x \in(A, B)-S .
\end{aligned}
$$

In the last equation a change of sign can occur only at a root of $g(x)$ of even multiplicity. More is true. Let $(a, b)$ be a component of $(A, B)-S$, with $a \in S$, $b \in S$. Then $u(a)=f(a), u(x)>f(x)$ if $x>a$ and near $a$, so that $d u / d x>d f / d x$ for $x$ near and $>a$; hence we have + in (3.1). Near $b$ for $x<b$ we have similarly $d f / d x>d u / d x$, hence the sign - in (3.1). Hence there lies between $a$ and $b$ a root of $g(x)$ of even multiplicity. Moreover, the set $\{x: x \in(A, B), u(x)$ $=f(x)\}$ exceeds $S$ by no more than finitely many points.

Remark. Theorem 3.1 remains true if the straight arc $[A, B]$ is replaced by any analytic Jordan arc $J$. To see this let $\omega: J \rightarrow[A, B]$ be an analytic diffeomorphism. Put for any Borel set $E$ of $J, \mu(E)=\nu(\omega(E))$. With $S \subset J$, we have 


$$
\begin{aligned}
\int_{S}-\log |x-y| d \mu(y)= & \int_{\omega(S)}-\log |\omega(x)-y| d \nu(y) \\
& +\int_{S}-\log \frac{|x-y|}{|\omega(x)-\omega(y)|} d \mu(y) .
\end{aligned}
$$

It is readily verified that the second integral is an analytic function of $x$ for $x \in J$, hence of $\omega(x) \in[A, B]$. Thus the first integral on the right satisfies the conditions of the theorem as function of $\omega(x)$, and $\omega(S)$ is a union of finitely many disjoint intervals. The same is then true for $S \subset J$.

4. A case where Theorem 3.1 applies is furnished by

Theorem 4.1. Let $\Omega$ be a smooth bounded domain of $\boldsymbol{R}^{2}$, and the interval $[A, B]$ of an $\boldsymbol{R}^{1}$ axis be $\subset \Omega$. Let $\psi(x), x \in[A, B]$, be real and analytic, and denote by $h(z)$ the smallest superharmonic in $\Omega$, continuous in $\Omega \cup \partial \Omega, \geq 0$ on $\partial \Omega$ and such that $h(x) \geq \psi(x)$, if $x \in(A, B)$. Assume that $\psi(x) \leq 0$ outside a compact subset of $(A, B)$, but not identically $\leq 0$ on $(A, B)$. Write $h(z)=$ $\int_{S} G(z, y) d \mu(y)$, where $G$ is Green's function of $\Omega$ and $\mu$ is a measure of compact support $S \subset(A, B)$. Then $S$ is the union of finitely many disjoint intervals.

Proof. $G\left(z, z^{\prime}\right)=-\log \left|z-z^{\prime}\right|+\gamma\left(z, z^{\prime}\right)$, where $\gamma$ is analytic for $z, z^{\prime} \in \Omega$. Therefore

$$
u(z)=h(z)-\int_{S} \gamma(z, y) d \mu(y)=\int_{S}-\log |z-y| d \mu(y)
$$

has the property:

$$
\begin{gathered}
u(x) \geq \psi(x)-\int_{S} \gamma(x, y) d \mu(y) \equiv f(x), \quad x \in(A, B), \\
u(x)=f(x), \quad x \in S,
\end{gathered}
$$

with $f(x)$ analytic in $[A, B]$, and hence Theorem 3.1 applies.

\section{References}

[1] Hans Lewy, On a refinement of Evans' law in potential theory, Atti Acad. Naz. Lincei Rend. Cl. Sci. Fis. Mat. Natur. 48 (1970) 1-9.

Univesity of California, Berkeley 
